\title{
Strigolactones Biosynthesis and Their Role in Abiotic Stress Resilience in Plants: A Critical Review
}

\author{
Wajeeha Saeed, Saadia Naseem and Zahid Ali* \\ Department of Biosciences, COMSATS Institute of Information Technology, Islamabad, Pakistan
}

Strigolactones (SLS), being a new class of plant hormones, play regulatory roles against abiotic stresses in plants. There are multiple hormonal response pathways, which are adapted by the plants to overcome these stressful environmental constraints to reduce the negative impact on overall crop plant productivity. Genetic modulation of the SLS could also be applied as a potential approach in this regard. However, endogenous plant hormones play central roles in adaptation to changing environmental conditions, by mediating growth, development, nutrient allocation, and source/sink transitions. In addition, the hormonal interactions can fine-tune the plant response and determine

OPEN ACCESS

Edited by:

Benoit Schoefs,

University of Maine, France

Reviewed by:

Soulaiman Sakr

Agrocampus Ouest, France

Frantisek Baluska,

University of Bonn, Germany

*Correspondence:

Zahid Ali

zahidali@comsats.edu.pk

Specialty section:

This article was submitted to Plant Physiology,

a section of the journal

Frontiers in Plant Science

Received: 11 May 2017 Accepted: 10 August 2017 Published: 28 August 2017

Citation:

Saeed W, Naseem S and Ali Z

(2017) Strigolactones Biosynthesis and Their Role in Abiotic Stress Resilience in Plants: A Critical Review.

Front. Plant Sci. 8:1487.

doi: 10.3389/fpls.2017.01487 plant architecture in response to environmental stimuli such as nutrient deprivation and canopy shade. Considerable advancements and new insights into SLs biosynthesis, signaling and transport has been unleashed since the initial discovery. In this review we present basic overview of SL biosynthesis and perception with a detailed discussion on our present understanding of SLS and their critical role to tolerate environmental constraints. The SLs and abscisic acid interplay during the abiotic stresses is particularly highlighted.

Main Conclusion: More than shoot branching Strigolactones have uttermost capacity to harmonize stress resilience.

Keywords: Strigolactones, abscisic acid, abiotic stress, crosstalk, phytohormones

\section{INTRODUCTION}

Strigolactones (SLs) were initially known as host-derived germination stimulants for parasitic weeds from the genera Striga and Orobanche. Later on, SLs were found to be host-detection and hyphal-branching signals for arbuscular mycorrhizal (AM) fungi (Akiyama et al., 2005; Besserer et al., 2006). In addition to their original, dual role as signaling molecules in the rhizosphere, SL were further demonstrated to be a new class of branch-inhibiting phytohormones, which regulate the overall architecture of land plants (Gomez-Roldan et al., 2008; Umehara et al., 2008). This benchmark discovery in SL research led to the exponential growth of investigations on the topic in the past 8 years, which in turn opened a Pandora box of findings on biological and molecular aspects of SL activity; as more and more research groups become interested in the biological and physiological role of SL, additional functions are likely to be identified in the future. A number of authoritative reviews have appeared in the past few years, specifically covering the functions of SL 
in plant development and interactions with root symbionts and parasitic weeds (Ruyter-Spira et al., 2013; Waldie et al., 2014; Al-Babili and Bouwmeester, 2015; Zhang et al., 2015). Here, after a short summary of our current understanding of their biosynthesis and perception, we wish to rather report on recent breakthroughs and novel avenues of research in the area of SL functions in plant resilience and resource allocation under abiotic stress, especially emphasizing the organ-specific cross-talk between SL and abscisic acid (ABA) under drought.

\section{SHORT OVERVIEW OF SL BIOSYNTHESIS AND PERCEPTION}

\section{Biosynthesis}

More than 20 SL and SL-like compounds have been identified so far in the root exudates of several plant species. They all share a conserved tricyclic lactone structure containing rings referred to as $\mathrm{ABC}$ rings, linked via an enol-ether bridge to an invariable $\alpha, \beta$-unsaturated furanone moiety named $\mathrm{D}$ ring. The bioactiphore resides within the region that connects the D-ring to the core; chemical diversity is given by the stereochemistry of the $\mathrm{B}-\mathrm{C}$ ring junction, the size of the $\mathrm{A}$ ring, and the substitution patterns of the $\mathrm{A}$ and $\mathrm{B}$ rings reviewed by (AlBabili and Bouwmeester, 2015) see Figure 1 for some exemplary structures. Rather wide collections of branching mutants are available in Arabidopsis thaliana [known as more axillary growth (max) mutants], Oryza sativa [dwarf (d) or high-tillering dwarf (htd) mutants], Pisum sativum [ramosus (rms) mutants] and Petunia hybrida [decreased apical dominance (dad) mutants]: their analysis was instrumental to understand and define most of the current assembly of SL biosynthetic (and signaling) pathways. Based on the observation that plants treated with inhibitors of carotenoid biosynthesis exude fewer SL from their roots, a hypothetical SL-biosynthetic pathway was initially proposed, with $\beta$-carotene as a substrate for carotenoid-cleavage dioxygenase (CCD) enzymes (Matusova et al., 2005). CCDs specifically cleave double bonds in carotenoid molecules to form carbonyl compounds called apocarotenoids (Auldridge et al., 2006). Later on, it was proven that indeed two related CCDs, CCD7 and CCD8, act sequentially in the pathway (being known, respectively, as D17/HTD1 and D10 in rice; RMS5, RMS1 in pea, $D A D 3, D A D 1$ in petunia, $M A X 3, M A X 4$ in Arabidopsis) (Morris et al., 2001; Sorefan et al., 2003; Booker et al., 2004, 2005; Snowden et al., 2005; Arite et al., 2007). Rice DWARF27 (D27) and its ortholog in Arabidopsis ATD27, an iron-binding, plastidlocalized $\beta$-carotene isomerase, works upstream of CCD7 and CCD8 catalyzing the conversion of all-trans- $\beta$-carotene into 9cis- $\beta$-carotene $(\mathrm{C}-40)$. The latter acts as substrate for CCD7 to cleave cis-configured carotenoids into 9-cis- $\beta$-apo-10'-carotenal (C-27) and $\beta$-ionone (C-13) (Schwartz et al., 2004; Alder et al., 2012; Waters et al., 2012a). CCD8 then acts on the C27 product of enzymatic cleavage to form a SL-like compound named Carlactone (CL), which is an intermediated compound in the SL pathway containing only $\mathrm{A}$ and $\mathrm{D}$ rings with enol ether bridge (Alder et al., 2012). CL act as endogenous precursor for more specific SLs shown in Figure 1, and exhibit SL like properties by inhibition of shoot branching in SL biosynthetic mutants (Rice, Arabidopsis) and promotion of seed germination Striga hermonthica (Scaffidi et al., 2013; Abe et al., 2014; Seto et al., 2014). The genes acting downstream to CCD8 include MAX1 in Arabidopsis, which encodes a cytochrome P450 of the CYP711A1clade and is responsible for the conversion of CL into functional SLs such as 5-deoxystrigol (Stirnberg et al., 2002; Booker et al., 2004; Alder et al., 2012). MAX1 putatively converts CL into functional SL by rearrangements and modifications (hydroxylation, oxidation), converting CL to carlactonic acid (CLA) then further transformed to methyl carlactonoate (MeCLA) by an unknown enzyme (Abe et al., 2014). The latter, at least in Arabidopsis, is perceived by the SL-binding moiety of the SL receptor D14 (see Perception and Signal Transduction) and has SL-like activity, even though its carbon backbone does not fully meet the general SL structure requirements (Figure $\mathbf{1}$ ).

In rice, one MAX1 paralogue converts carlactone into ent-2'epi-5-deoxystrigol, the presumed precursor of rice SL. A protein encoded by a second MAX1 homolog then catalyzes the conversion of ent-2'-epi-5-deoxystrigol to orobanchol (Zhang et al., 2014), thus explaining some of the basic chemical diversity of SL (the stereochemistry at the BC-ring junction), at least in rice. Further investigation of MAX1 and of its orthologs in Arabidopsis and rice revealed that this gene is expressed in all vascular tissues, functions only in late steps of SL synthesis, and is responsible for at least some of the structural diversity of SL (Booker et al., 2005; Umehara et al., 2010; Xie, 2016). In vitro association of recombinant $M A X 1$ with $\mathrm{CL}$ in yeast microsomal system confirms that MAX1 acts as CL oxidase to convert CL stereo specifically into 9-desmethyl-9 carboxy-C2 or carlactonoic acid (CLA). Presence of both CLA and MeCLA (methyl ester carlactonoate) endogenously in Arabidopsis roots by LC-MS/MS also confirms that CL alone is the target of MAX1. Interestingly exogenous application of both CLA and MeCLA was found to rescue maxl mutant phenotype in Arabidopsis, however, only MeCLA act as substrate to bind with putative SL receptor of Arabidopsis thaliana DWARF14 (AtD14) (Abe et al., 2014). Elucidative observation were made on stereo selectivity of CL endogenously during interaction with MAX1 by labeling experiments in rice where chemically synthesized ${ }^{13} \mathrm{C}$ labeled $\mathrm{CL}$ was catalyzed to ${ }^{13} \mathrm{C}$ - 2 -epi-5DS and ${ }^{13} \mathrm{C}$-orobanchol (Abe et al., 2014; Zhang et al., 2014). More recently, another protein was reported to act downstream of $M A X 1$ in Arabidopsis. Lateral Branching Oxidoreductase (LBO) is an oxidoreductase-like

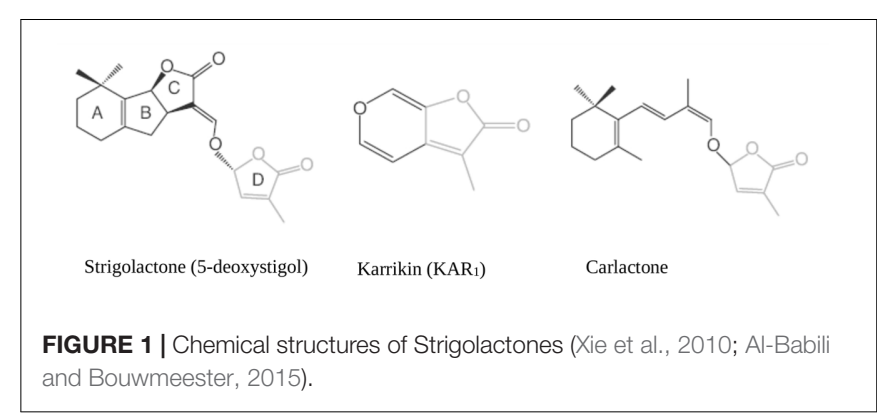


enzyme of the 2-oxoglutarate and Fe(II)-dependent dioxygenase superfamily, whose expression pattern partially overlaps with that of MAX3. LBO acts on MAX1 products, such as MeCLA or CLA and converts them into an unknown SL-like compound that is hypothesized to be more effective than SL and responsible for branching inhibition (Brewer et al., 2016). For more detailed and latest discoveries in SL biosynthesis see (Al-Babili and Bouwmeester, 2015; Koltai and Prandi, 2016).

\section{Perception and Signal Transduction}

Like most of the plant growth regulators (auxins, gibberellins, jasmonate), SL signaling mechanisms are executed by proteosomal degradation. The SL signaling machinery comprises at present the $\alpha / \beta$-fold hydrolase named (At)D14/DAD2/RMS3, the F-box leucine-rich protein MAX2/RMS4/D3, and the D53 a repressor protein, which holds some similarity to class I CIp ATPase enzymes and belongs to a small family of proteins [SMAX1-like (SMXL)] (Stanga et al., 2013). MAX2, leucine rich F-box proteins has been shown to be part of the SKP1-CUL1-F-box-protein (SCF)-type ubiquitin ligase complex, which ubiquitinates target proteins tagging them for proteosomal degradation (Stirnberg et al., 2007; Arite et al., 2009). Interestingly, components in the auxin, gibberellin, jasmonic acid and salicylic acid perception and signal transduction machinery share similarities with either D14, MAX2 or both. The auxin and jasmonic acid co-receptors TIR1 and COI1, for example, are F-box proteins; the gibberellin receptor GID1 and the Salicylic Acid-Binding protein SABP2 belong to the $\alpha / \beta$-hydrolase superfamily. In all of the above pathways, phytohormones act as molecular glues, allowing assembly of the active receptor/signaling complex and leading to proteasomal degradation of negative regulators of signaling (Dharmasiri et al., 2005; Ueguchi-Tanaka et al., 2007; Hamiaux et al., 2012). The current mechanistic hypothesis of SL perception implies that D14 acts in a signaling pathway mirroring one of gibberellins and including the F-box protein MAX2 (Seto and Yamaguchi, 2014). However, while GID1 does not modify its ligand, D14 is an active hydrolase; it is also a single-turnover enzyme, which explains its seemingly very low turn-over rates. The net result is that, upon binding, the hydrolysed ligand (in its bioactiphore moiety) and the receptor are locked together and thus unavailable for further perception events (de Saint Germain et al., 2016). D14 is also unique being receptor and enzyme at the same time unlike other phytohormones. D14-mediated perception of SLs depends on a catalytic triad (Ser, His, Asp) for binding and hydrolysis of SLs (Hamiaux et al., 2012). It has been proposed that MAX2/D3 acts in the SCF complex as recognition subunit for SL-loaded D14 and downstream repressors (see below). D14 in turn interacts with SLs and modifies them, mainly via its hydrophobic ligand-binding pocket and particularly the nucleophilic residues in the triad, which attack the $\mathrm{D}$ ring at the carbonyl of butenolide and thus separate it from the ABC part (Scaffidi et al., 2012; Zhao et al., 2013). The D14-SL complex is thought to change its conformation, which is mainly responsible for SL signal transduction. In vitro interaction GR24 has been shown to thermally destabilize D14 and for that catalytic triad is necessary. D14 loaded with SL is then recruited to $\mathrm{SCF}^{\mathrm{MAX} 2}$, which in turn directs further degradation of target proteins (e.g., D53) via the proteasome (Figure 3) (Jiang et al., 2013; Zhao et al., 2015). D14 itself is a target for late proteasomal degradation, which is thought to reinforce tissue desensitization to SL (Chevalier et al., 2014). It should be noted that the identity of D53 molecular partners is so far unknown, however, some orthologs have been reported in Arabidopsis (SUPPRESSOR OF MAX-2-LIKE 6-8 SMXL6-8) involved in repression of shoot branching and other SL regulated process (Soundappan et al., 2015); D53 because of the EAR motifs it contains, D53 is supposed to interact with the transcriptional corepressors termed topless-related (TPR) proteins. The D53-TPR complex could repress the transcription of targets of SL action (Jiang et al., 2013; Bennett and Leyser, 2014; Smith and Li, 2014), but this has not yet been demonstrated. Other members of the SMXL family might be in charge to modulate different aspects of SL action, while D53 seems to be the main repressor of the shoot branching effect of SL.

Several recent reports put forward interesting hypotheses on the evolution of ligand and signaling specificity by members of the wider family of D14 and D14-like proteins. The latter is a closely related protein clade to D14 proteins, some members of which have been characterized as receptors of host-exuded SL by parasitic plants, and would then represent a case of convergent evolution on SL perception with D14 (Toh et al., 2014; Conn and Nelson, 2015; Tsuchiya et al., 2015). This latter family comprises also proteins that were likely sub-functionalized to perceive other ligands in higher plants, such as karrikins - smokederived, D-lactone ring-containing compounds (Flematti et al., 2004; Waters et al., 2012b); both the perception of SL (in host and parasitic plants) and of karrikins require MAX2 (Zhao et al., 2015). How MAX2-mediated signaling can discriminate between different signaling pathway to generate different responses is still unknown; F-box proteins, however, are known to be promiscuous in target recruitment (Nelson et al., 2011; Nakamura et al., 2013), so the components of the signaling complex might be combinatorially assembled with different targets than D53, depending on the ligand and receptor moiety involved (Flematti et al., 2016). More aspects about SLs biosynthesis, perception, and signaling as well as structure-function relationships in the SL molecular family have been nicely addressed and updated in recent reviews (Ruyter-Spira et al., 2013; Al-Babili and Bouwmeester, 2015; Marzec, 2016; Bythell-Douglas et al., 2017; Waters et al., 2017).

\section{STRIGOLACTONES IN ABIOTIC STRESSES: HORMONAL INTERPLAY AND REGULATION}

The discovery of SLs provided new opportunities in the last decade to explore hormonal regulation of plant development and acclimatization to environmental constraints. These research endeavors also identified new instances of hormonal cross talk participating to the orchestration of overall responses in plants.

Hormonal cross talk is largely at work to allow an appropriate plant response to environmental stimuli, as well as changes in 


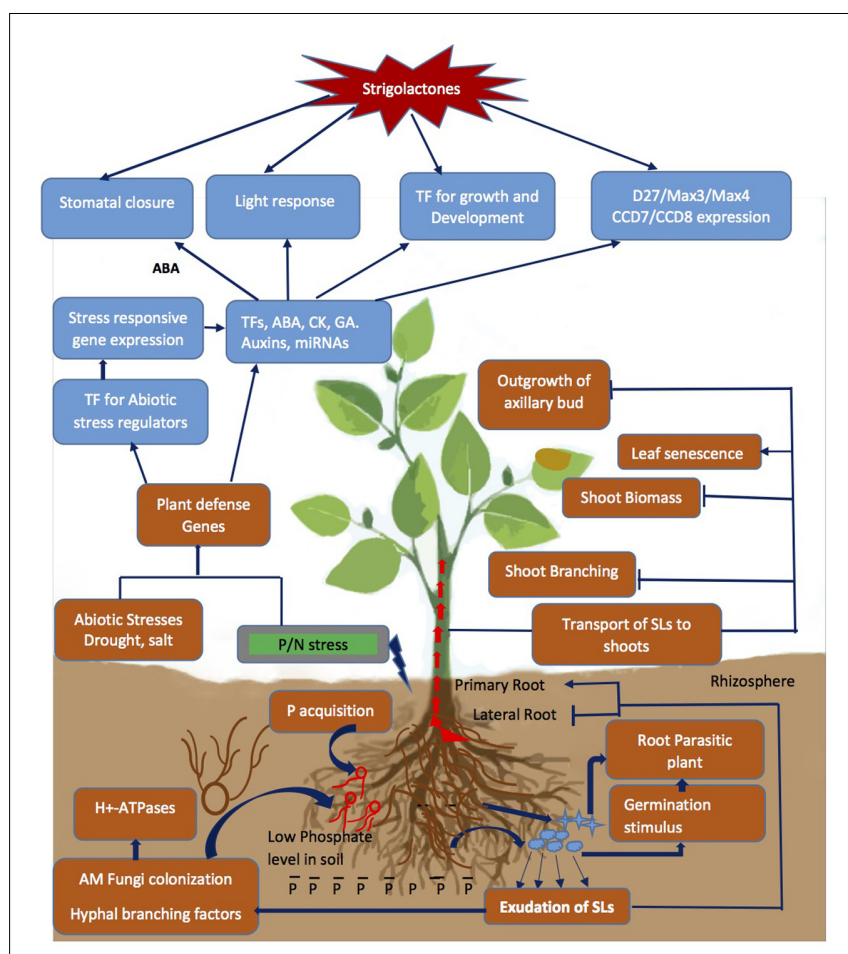

FIGURE 2 | Strigolactone (SL) mediated signal transduction and changes in physiological response due to Pi depletion and abiotic stresses encountered by the plant. The P starvation alone or combined with drought/salinity leads to cascade of event from exudation of SLs to changes in above/below ground architecture of plant by cross interactions with other phytohormones and regulation of stress responsive genes, TFs and miRNAs to modulate stress tolerance. Blue arrows represent the process promoted by the SLs and capped blue lines represents repression. architecture and acclimatization under challenging conditions such as nutrient starvation and heat/cold/salinity/light stress, by mediating growth, development, nutrient allocation and source/sink transitions.

Plastid-derived apocarotenoids produced in response to environmental conditions in plants include several plant growth regulators and signaling molecules besides SLs, such as retinoids and ABA. As mentioned in the Section "Introduction," SLs have diverse biological functions in addition to regulation of branching phenotype, being important signaling compounds in the rhizosphere (Figure 2). Knowledge of SLs biosynthesis and their physiological role in monitoring the architecture of plants led to well established fact that SLs, need to modulate and interact with many phytohormones - particularly auxins and ABA - to exert their effect.

Similar to other plant hormones and growth regulators, SL biosynthesis and activity is regulated by complex networks and cross talk with other hormones (Cheng et al., 2013), and recent findings suggest that SLs too, are key players of growth optimization in plants under sub-optimal environmental conditions.

The interactions among SLs and other phytohormones have been investigated in the past, especially as auxin is concerned.

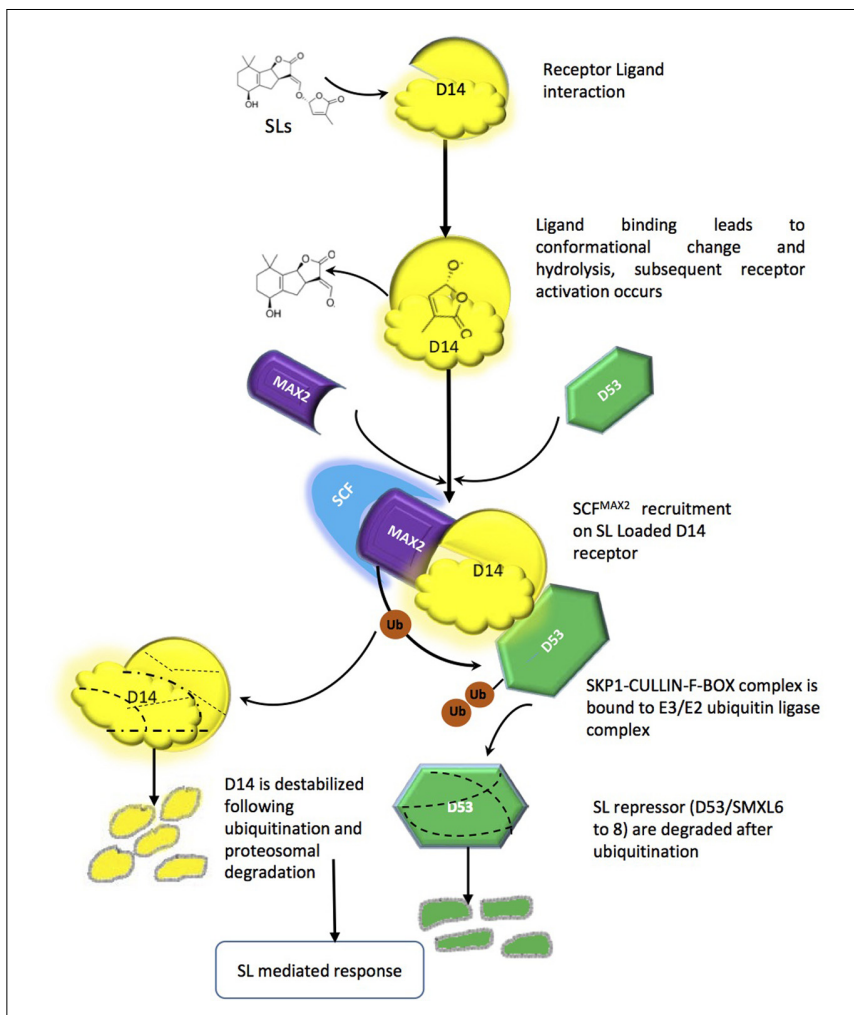

FIGURE 3 | Strigolactone perception and signaling mechanism, Interaction between the ligand-binding moiety of the SL receptor complex (the $\alpha / \beta$ hydrolase D14) and the co-receptor moiety (the F-box MAX2). Such interaction promotes further binding between MAX2 and its target(s), leading to ubiquitination and degradation of the latter by the proteasome machinery and downstream signaling pathways.
Auxin regulates SL biosynthesis and is involved in most of the SL-regulated developmental processes (Hayward et al., 2009; Crawford et al., 2010). For instance, SL mainly act as second messenger in shoot branching, by damping the transport of auxins in stem thereby inhibiting axillary bud outgrowth (Brewer et al., 2009). Another model for inhibition bud outgrowth proposed, SLs acts systemically to suppress PIN-FORMED (PIN) polar auxin transport protein from apical meristem thus limiting the bud growth (Bennett et al., 2006; Prusinkiewicz et al., 2009). Several lines of evidence support SL-Auxins interaction. Based on auxins and SL signaling regulatory effects on seminal root length, lateral root (LR) formation and root hair elongation (RH) became evident. Regulatory role of SLs on polarization and abundance of PIN protein in roots growth has also been indicated. In roots auxin signaling acts downstream to SLs (Brewer et al., 2009; Mayzlish-Gati et al., 2012; Sun et al., 2014). Exogenous application of synthetic auxins that are not secreted by efflux carriers (2,4-D) reversed GR24 mediated root effects in tomato while NAA and IAA failed to restore symmetric root growth and root hair (RH) elongation (Koltai et al., 2010). More recently SL biosynthetic and signaling mutants treated with exogenous GR24 showed that depletion of SL promotes lateral root formation. Both WT and biosynthetic mutants of SL in the 


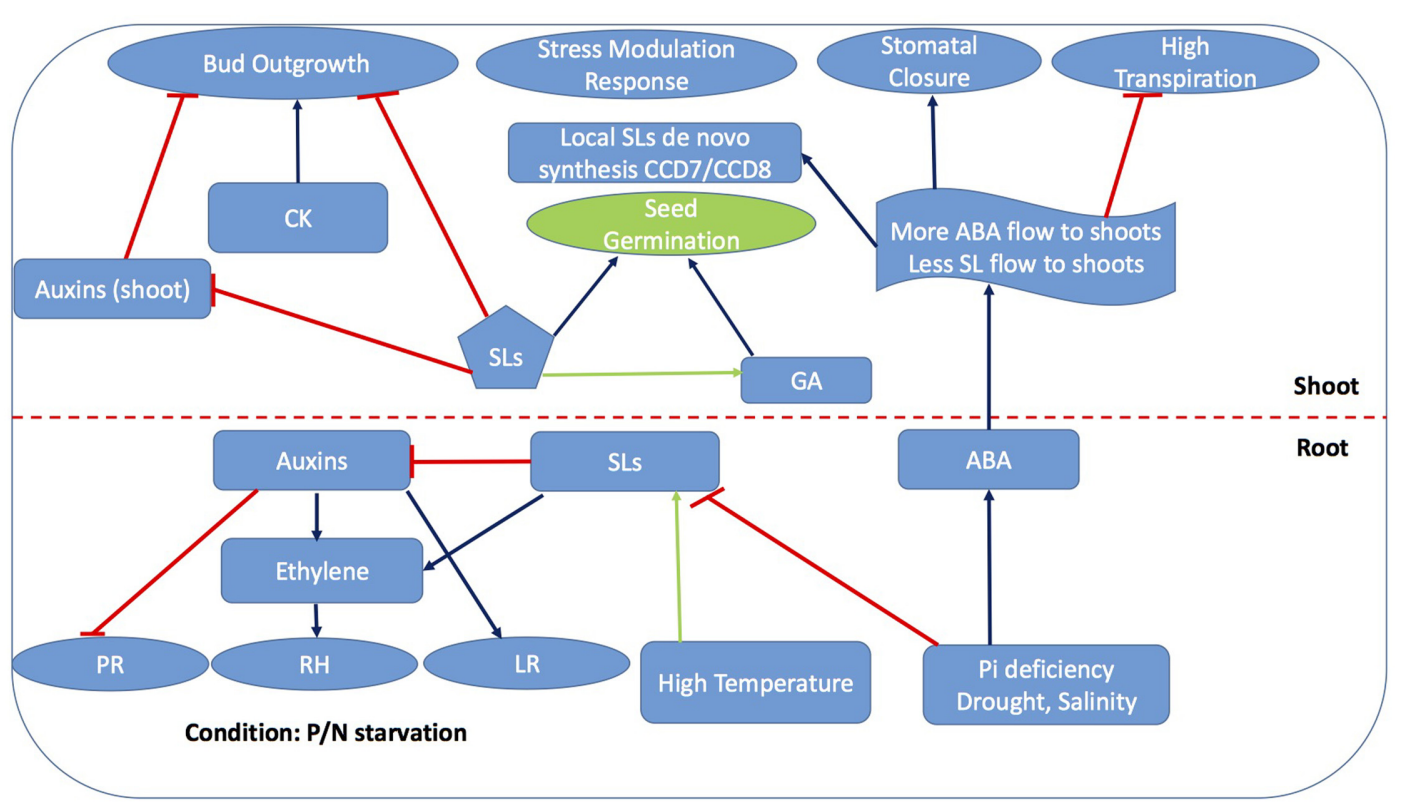

FIGURE 4 | Strigolactone-ABA cross linking platforms most of the phytohormones interactions for stress mediated response. Blue arrows represent activation of process while capped red lines represent repression while green arrows represent partially explored process promoted by SLs. PR, Primary roots; RH, Root hair; LR, Lateral roots; GA, Gibberellic acid; CK, Cytokinins

presence of GR24 showed limited lateral root growth, however, this phenotype wasn't evident in max2 mutants (Kapulnik et al., 2011). In most plants SL biosynthetic genes (MAX3 and MAX4) were upregulated by exogenous auxins application (Foo et al., 2005; Johnson et al., 2006; Arite et al., 2007). Role of MAX2 in auxin interaction has also been proposed under Pi deficiency, which leads to PIN2 depletion in MAX2 dependent manner. These changes in auxin efflux in SL/MAX2 dependent manner are of prime importance in changing the root architecture like increased density and length of root hair and promotion of lateral root growth due to nutrient depletion. These data suggest that SL manipulate and regulate auxins pathway either by dampening its transport or regulate perception by promoting transcription of auxins receptors TIRI reviewed by (Koltai, 2015).

Interaction of SLs with other phytohormones such as salicylic acid (SA), cytokinins (CK), gibberellins (GA), ABA have also been explored in some detail (Wallner et al., 2016). Particularly role of SL in CK and auxins in apical dominance as well as root development is emerging ( $\mathrm{Xu}$ et al., 2015), The mechanisms of cross talk between SLs and other phytohormones (Figure 5) could be in the alteration in biosynthesis, sensitivity, and/or transport of either hormone (Cheng et al., 2013), whose study offers chances of better understanding hormonal regulation of plant physiology under stress. Advancements in SL based research collectively implies the hormonal interaction responsible for fine tuning the plant response, thereby emphasizing the regulatory role of SL, CK, auxins and ABA during sub-optimal environmental conditions. In the following sections, we will focus on the regulation of SL biosynthesis under abiotic stress, and on SL crosstalk with other phytohormones (particularly ABA) during abiotic stress.

\section{SL Production under Nutrient Starvation}

Phosphate (P) and/or Nitrogen (N) deficiency in the soil is a serious abiotic source of stress and commonly encountered by most land plants. The only accessible form of $\mathrm{P}$ for plants in soil is inorganic (Pi) (Péret et al., 2011). Since the prime site for $\mathrm{Pi}$ acquisition is the roots, critical changes in plant architecture occur below ground during Pi deficiency among them are increased root growth and reduced shoot/root ratio, inhibition of shoot branching, limited primary root elongation along with extensive growth of lateral roots and root hairs (Linkohr et al., 2002; Niu et al., 2013). Consistent with the role of SLs in recruitment of AM fungi, their release and production is promoted by low soil P level (Jamil et al., 2014). Yoneyama et al. (2007a) studied orobanchol exudation form red clover (Trifolium pratense L.) in response to deficiency of various nutrients ( $\mathrm{P}, \mathrm{N}, \mathrm{K}, \mathrm{Ca}$, and $\mathrm{Mg}$ ); later on, they also tested the effects of $\mathrm{N}$ and $\mathrm{P}$ deprivation on deoxystrigol levels in sorghum (Sorghum bicolor [L]. Moench) exudates (Yoneyama et al., 2007b). SL quantification by LC-MS/MS showed a 20-fold increase of orobanchol in red clover exudates obtained under $\mathrm{P}$ starvation, while in sorghum, a 30-fold higher level of the major SL 5-deoxystrigol was reported under low $\mathrm{P}$ and also N. After this seminal work, production and release of SLs under $\mathrm{P}$ and/or $\mathrm{N}$ deficiency were tested in different leguminous/non-leguminous species. Most of the plants exuded more SLs in response to both $\mathrm{P}$ and $\mathrm{N}$ deficiency except tomato (Solanum lycopersicum $\mathrm{L}$ ) and alfalfa (Medicago sativa), which only responded to $\mathrm{P}$ 
starvation (Yoneyama et al., 2007b). As more legumes and nonlegumes were tested, it became clear that the ability to associate with root-nodulating, $\mathrm{N}$-fixing bacteria does not correlate strictly with the response to $\mathrm{N}$ deficiency in terms of SL exudation. Indeed, most legumes (red clover, alfalfa, and crimson clover [T. incarnatum L.]) show increased SL exudation under P deficiency only, while most non-legumes respond to deficiency of both $\mathrm{N}$ and $\mathrm{P}$; however, exceptions exist in both groups, as the legume Chinese milk vetch (Astragalus sinicus L.) responds to both (Yoneyama et al., 2012) while the non-legume tomato reacts only to P deficiency (López-Ráez et al., 2008a). Therefore, the ecological implications of increased vs. stable SL exudation under $\mathrm{N}$ deficiency in different species became unclear, even though it is now proven that SL are needed for a full nodulating response (Soto et al., 2010; Foo et al., 2013; Liu et al., 2013; De Cuyper et al., 2015), and are also able to induce surface motility (swarming) in N-fixing bacteria. It was then suggested that decreasing shoot $\mathrm{P}$ levels are the real triggers for SL induction under nutrient starvation; as $\mathrm{N}$ starvation affects shoot $\mathrm{P}$ levels in some but not all species equally, the effects of $\mathrm{N}$ deficiency on SL induction will vary depending widely on the type of plant, type of nutrient, degree of nutrient stress and macronutrient uptake strategies (Yoneyama et al., 2012, 2013). Now the fact is well documented that SLs mediated stress adaptive response are generated as a result of nutrient deficiency (Umehara et al., 2010; Jamil et al., 2011). Furthermore, while plants produce blends of SL molecules, only one or two specific types are induced under specific nutrient starvation conditions (Yoneyama et al., 2008). The ecological meaning of this selectivity is currently unknown, as remains unexplored the possibility that different SL blends have different signaling "meaning" for the producing organism and/or for the soil biota.

\section{SL Signaling Is Responsible for Some Plant Responses to P/N Starvation Stress}

While the increased release of SLs in response to limiting $\mathrm{P}$ and/or $\mathrm{N}$ in soil has been described in many species, questions regarding the mechanisms of stress signaling leading to higher expression of SL biosynthetic proteins are still open. Umehara et al. (2015) found that rice genes involved in biosynthesis and perception $(D 10, D 17, D 27)$ of SLs were differentially regulated by $\mathrm{P}$ starvation/supplementation; additionally, the transcript encoding PhPRD1, the ABCG transporter responsible for exudation in soil and translocation of SLs from roots to shoot in petunia (Petunia hybrida) also accumulates during P starvation (Yoneyama et al., 2007a; Péret et al., 2011; Kretzschmar et al., 2012). This allows to assume that in response to low $P$ level, the transport of SLs from the roots to the shoot increases and thus, not only root but also shoot architecture may be affected. Indeed, an increase in roots exudated orobanchol as well as the inhibition of lateral bud outgrowth was reported in WT Arabidopsis under P starvation (Umehara et al., 2010; Mayzlish-Gati et al., 2012; Yoneyama et al., 2012), which is in accordance with previous findings in rice (Jamil et al., 2011). Being Arabidopsis a nonhost to AM fungi, it is hypothesized that - as it is not to the purpose of luring AM toward the P-depleted root - such increase in SLs may help to modify the architecture of plant (roots) for better utilization of available Pi see for example (Ito et al., 2015). Transcriptome analysis in alfalfa under nutrient stress also showed upregulation of 189 genes associated with AM fungal colonization/P/N deficiency, and those included SLbiosynthetic genes (Bonneau et al., 2013). Taken together, all available data point to the activation of SL metabolism under low $\mathrm{Pi}$, leading to changes in nutrient allocation by inhibition of shoot branching as a survival and acclimatization strategy. At the same time, root morphology also changes under P starvation, by an increase in lateral RL and repression of PR growth. Moreover, root hair density and length increase, allowing for a larger soilroot interface and thus a more efficient Pi uptake (Figure 2). In general, SLs affect those features of root architecture (seminal $\mathrm{RL}$, lateral root formation, RH elongation) controlled by auxins during P starvation (Pérez-Torres et al., 2008; Péret et al., 2014). SL-mediated control of root architecture during Pi depletion requires MAX2 (Mayzlish-Gati et al., 2012). Consequently, under low Pi and $\mathrm{N}$ conditions, SL signaling initiates morphological changes in Arabidopsis, leading to altered expression of SL biosynthetic genes (MAX3, MAX4), RH elongation, activation of $\mathrm{P}$ transporter genes, regulation of phosphate starvation marker genes (PSI), anthocyanin accumulation and reduction in fresh weight of plant (Sun et al., 2014; Ito et al., 2016).

\section{SLs and ABA Interplay}

Although $\mathrm{ABA}$ is the most studied stress-responsive hormone, the individual role of ethylene, CKs, BRs, and auxins during environmental stress is emerging, as is the impact of their mutual cross-talk (Fujita et al., 2011; Arc et al., 2013; Fahad et al., 2015; Wani et al., 2016). SLs as well, were recently shown to play a prominent role in abiotic stress responses (see SL Signaling is Responsible for Some Plant Responses to P/N Starvation Stress and below), and we have thus entered a new phase in which their interaction with other phytohormones in the frame of abiotic stress resistance is being targeted experimentally. $\mathrm{ABA}$ is sometimes referred to as the stress responsive growth regulator par excellence, due to its role in stomatal closure and, in some plants, as long-range signal triggered by abiotic stresses like drought, desiccation, salinity, pathogen attack and wounding (Zeevaart and Creelman, 1988; Davies et al., 2002). Changes in ABA levels induce expression of many genes either directly or indirectly by upregulation/downregulation of transcription factors (Chandler and Robertsonz, 2003). During dehydration indeed, ABA accumulation in guard cells leads ultimately to stomatal closure. De novo synthesis of ABA in stressed leaves and roots is also reported (Boursiac et al., 2013). Changes in level of $\mathrm{ABA}$ also takes place via influx through transporters, Reactive oxygen species (inactivate conjugates). Peculiar increase in ABA accumulation in guard cell are mainly accounted for dehydration stress related rapid response, while long term soil water deficit and drought conditions are circumvented by ABA synthesis in main vasculature (vascular parenchyma, plastid, cytosol) (Merilo et al., 2015). These sudden changes in ABA levels plus its ability to transport over long distance peculiarly act as stress messenger. This also involve changes in long distance transport of $\mathrm{ABA}$ as 
well as modulation of ABA in guard cell leading to cascade of reactions for stomatal closure, reduce leaf expansion, promotion of root growth and prevent loss of water (Hong et al., 2013). Apart from stomatal closure as a mean to cope with stress, changes in $\mathrm{ABA}$ level also trigger transcriptional activation of genes encoding protein required for stress tolerance in plants, these include osmoprotectants, dehydrins, salinity and drought related genes (Wilkinson and Davies, 2002; Fujita et al., 2011).

Strigolactones have emerged to be key players in plant physiology under stress such as upon nutrient starvation (see SL Signaling is Responsible for Some Plant Responses to P/N Starvation Stress) but also drought, salinity (Liu et al., 2013; Bu et al., 2014; Ha et al., 2014; Visentin et al., 2016), and light stress (Gonzalez-Perez et al., 2011; Jia et al., 2014). ABA accumulates in plants due to the activity of enzymes in the 9-cis-epoxycarotenoid dioxygenase family (NCED), catalyzing the cleavage of 9-cisepoxycarotenoids to xanthoxin, i.e., the first step in the ABA biosynthetic pathway (Cutler and Krochko, 1999; Bouwmeester et al., 2007). Since the beginning of SL research, the shared carotenoid precursor of SLs and ABA have intrigued the possible crosstalk of SLs at various level of biosynthesis which have been partly explored (Figures 4, 5). Although the initial working hypothesis was that both hormones interact with each other at the biosynthetic level, and that induction of ABA biosynthesis impacts SLs formation and vice versa by substrate competition, recent work has highlighted more subtle interactions.

Root extracts and exudates of ABA-deficient maize plants having null mutations in the ABA-biosynthetic gene ZmNCED1 induced significantly less germination of the parasitic seeds, an effect that may be associated with low levels of SLs (Matusova et al., 2005; López-Ráez et al., 2008b). In a more recent report, $\mathrm{ABA}$ was shown to induce MAX3 and MAX4 transcript accumulation in Arabidopsis (Ha et al., 2014). These results collectively highlight a regulatory role for $\mathrm{ABA}$ in SL production, disfavouring the hypothesis of a direct involvement of NCEDs in SL production and of course, of the carotenoid substrate being limiting for the two competing pathways. Since SLs are also involved in AM fungal colonization and involvement of $\mathrm{ABA}$ in $\mathrm{AM}$ colonization has also been extensively investigated, the SL-ABA crosstalk may have implications in this area of research, too. Aroca et al. (2008) reported that lettuce plants colonized by AM fungi can modulate their endogenous $\mathrm{ABA}$ so to better cope with drought stress than non-mycorrhized plants. In tomato, ABA-deficient sitiens mutants having only $8 \%$ $\mathrm{ABA}$ as compared to wild-type, were less susceptible than the latter to AM fungal infection (Herde et al., 1999). Endogenous $\mathrm{ABA}$ is reported to enhance the spread of fungi as well as arbuscule development in tomato, while exogenous $\mathrm{ABA}$ treatment increases the rate of colonization (Herrera-Medina et al., 2007). These reports therefore suggest a role for ABA in controlling colonization by AM fungi, which may depend on underlying interactions with other plant hormones. For example, it is well established that $\mathrm{ABA} / \mathrm{GA}$ interaction with regulate various growth and developmental processes including AM colonization in tomato (Martín-Rodríguez et al., 2016). Since SL signaling mechanism has revealed surprising overlapping mechanism with GA pathway (Wallner et al., 2016). In such an interlinked context, the effects of ABA and SLs on AM fungi infection cannot be easily made apart (López-Ráez, 2016). The reduction of SL levels in the above-mentioned tomato mutants sitiens deficient in ABA could be the reason of low AM colonization; however, CCD8-silenced transgenic tomato was not significantly affected in AM colonization, indicating that the residual SL production in such lines might be enough to warrant efficient host perception by AM fungi (Kohlen et al., 2012). Also, there are hints - e.g., in lettuce - that not only AM colonization is affected by ABA and SLs, but that levels of the latter can be altered by AM colonization, also in dependence of water availability in soil (Aroca et al., 2013; Ruiz-Lozano et al., 2016).

A separate analysis should be dedicated to the converse effect i.e., of SLs on ABA levels - and to the SL-ABA cross-talk under abiotic stresses. In the first report of SL-ABA interaction by López-Ráez et al. (2010), blocking ABA synthesis by specific NCEDs inhibitors reduced the synthesis of 3 major SLs in tomato when compared to untreated wild type, along with a slight reduction of $\mathrm{ABA}$ in roots. Interestingly, tomato plants treated with an inhibitor of CCDs (among which, the SL biosynthetic enzymes CCD7 and CCD8 had unchanged ABA concentrations in roots, suggesting that while $A B A$ positively regulates $S L$ content, the opposite may not be true (López-Ráez et al., 2010). However, under drought or combined osmotic/low P stress, SLdeficient tomato or Lotus plants show significantly reduced ABA levels in their shoots (Liu et al., 2015; Visentin et al., 2016). In a following report by Ha et al. (2014), Arabidopsis SL biosynthetic and signaling max mutants were subjected to drought and salinity stress to investigate the involvement of SLs in abiotic stress resilience. No significant differences in ABA levels could be detected between wild-type and SL mutants in this species, but stressed tissues were not analyzed; this and the previous datasets on tomato and Lotus collectively suggest a general positive influence of SLs on ABA levels under stress, possibly with some species-specific variability. Nonetheless, both SL-deficient and perception mutants of Arabidopsis were reported as more stresssensitive at different developmental stages in the same work. The positive role of SL in abiotic stress resistance was further proven by exogenous application of SL at the shoot level, which improved performances under stress both of wild-type and of SLdeficient mutants, but not of SL signaling mutants. Interestingly, all SL mutants were partially insensitive to exogenous ABA as compared to wild-type plants, both at germination and seedling developmental stage (Ha et al., 2014). Sensitivity of the signaling mutant max2 in Arabidopsis to abiotic stresses as reported by Ha et al. (2014) was somewhat confirmed by Bu et al. (2014), whose conclusion on hypersensitivity of SL signaling mutants to dehydration and elevated transpiration rate due to reduced stomatal closure as compared to wild-type are in agreement with the above results. However, besides this shared conclusion, the two reports contradict each other at various levels. On one hand, Ha et al. (2014) reported that exogenous ABA negatively affects seed germination and development of wild-type seedlings but not of the SL mutants $\max 3$, max4 and $\max 2$. On the other hand, max2 displayed hypersensitivity to ABA at preand post-germination stages in the work by Bu et al. (2014). 


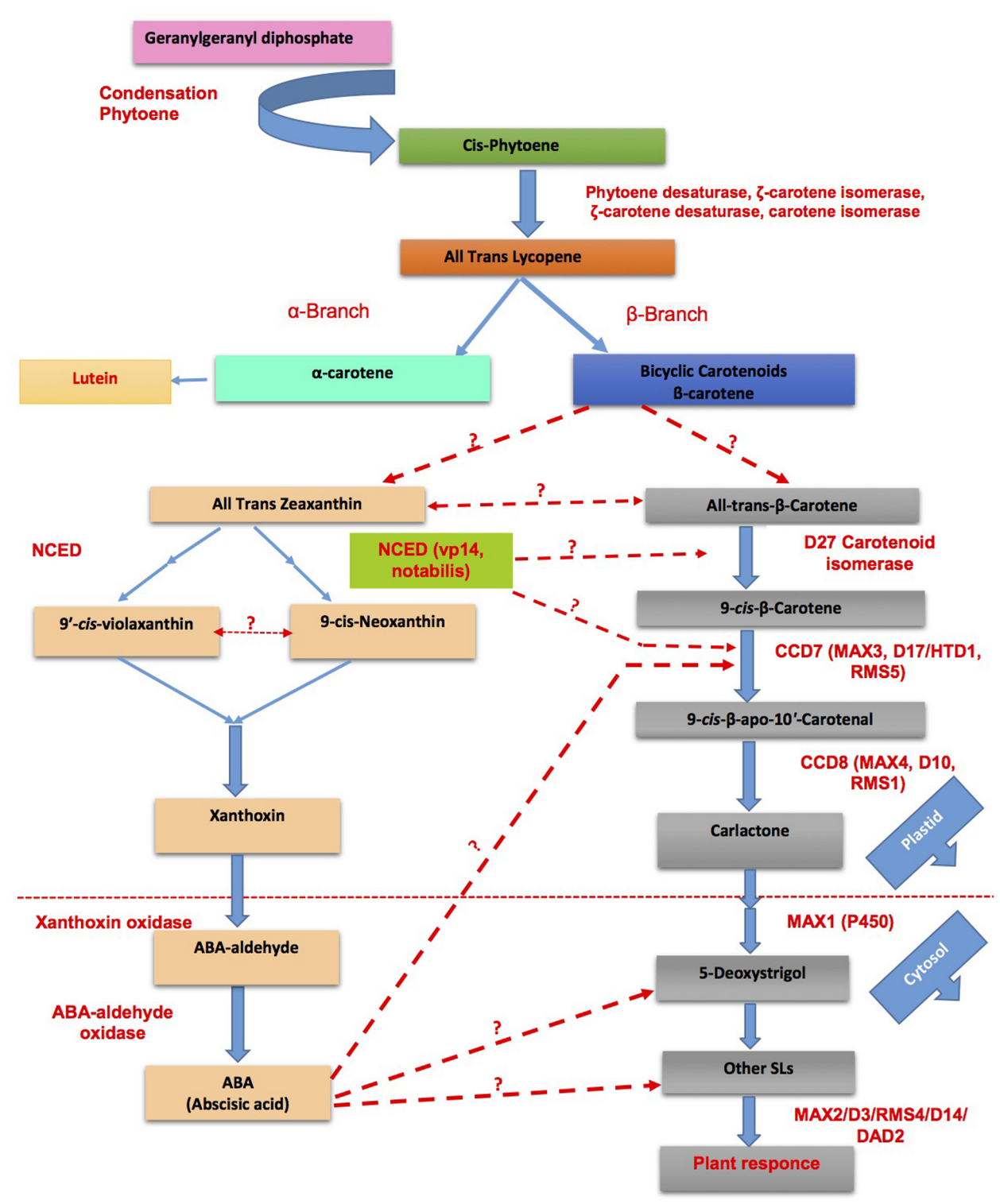

FIGURE 5 | Cross talk between SLs and abscisic acid (ABA) biosynthesis for the adaptability of plants in response to challenging environmental conditions (modified from Ruyter-Spira et al., 2013; Zhang et al., 2013). Dotted lines represent the unexplored/partially explored interactions.

More strikingly, SL-depleted Arabidopsis plants were reported as hypersensitive to stress in $\mathrm{Ha}$ et al. (2014), while Bu et al. (2014) found a similar phenotype only in the signaling mutant max2, thus excluding a direct role for the SL metabolites in osmotic stress responses. However, the contribution of SLs in osmotic stress resistance was confirmed in other species, thus supporting the results by Ha et al. (2014) in Arabidopsis. Liu et al. (2015) reported that SL-depleted Lotus japonicus was more stresssensitive than its wild-type counterpart; more recently, the same was proven in tomato (Visentin et al., 2016). In all three species, the effect was linked to partial insensitivity to endogenous and exogenous ABA. These datasets certainly show that endogenous SLs give an important positive contribution to stress resistance by increasing ABA sensitivity. This, coupled to lower ABA contents than in the wild-type, would certainly contribute to poor performances of SL mutants under drought. Altogether then, the drought-hypersensitive, ABA-hyposensitive phenotype shown by SL-depleted plants (tomato, Lotus, Arabidopsis) is persuasive proof that during stress, proper ABA accumulation and functioning at the guard cell level requires intact SL metabolism and signaling.

If SLs contribute to drought resistance one would expect their levels to increase under stress. This may be true in shoots, where although metabolites remain under the detection threshold, the transcript of biosynthetic genes are more concentrated in dehydrated than unstressed wild-type tissues, both in Arabidopsis 
and tomato (Ha et al., 2014; Visentin et al., 2016). However, surprisingly, the opposite is true for the main site of SL production under normal conditions, i.e., the roots. There, both the transcript of genes involved in SL biosynthesis and exudation, and the metabolites themselves in tissues and exudates, were markedly decreased by drought or salinity in nonmycorrhized tomato (Ruiz-Lozano et al., 2016; Visentin et al., 2016) and by osmotic stress in Lotus (Liu et al., 2015). In the latter set of experiments, even the increase of SL exudation triggered by $\mathrm{P}$ starvation alone was reversed to a sharp decrease under combined osmotic/low-P stress, indicating that in the case of multiple stresses, response to one can override the other.

The above-mentioned organ-specific dynamics of SL production during dehydration were investigated into more detail in tomato. The hypothesis of a possible role for the drought-triggered SL decrease at the root level in long-distance signaling of stress to the shoots was tested, by comparing the eco-physiological performances and ABA content of wild-type and SL-depleted plants with those of plants obtained by grafting wild-type shoots on SL-depleted roots, in a drought time-course (Visentin et al., 2016). This latter genotype combination was meant to mimic, as far as SLs are concerned, the hormonal balance typical of stress even in unstressed conditions - i.e., low SLs in the roots but normal SL metabolism in the shoot. The results show that under some respects (low gas exchange rates and high transcription of SL-biosynthetic genes in the leaves), such hetero-grafted plants behave as if they were indeed under mild stress, and thus, that low SL levels in the roots can, alone, signal distress at the root level. ABA content is, however, either unchanged or decreased in wild-type shoots grafted onto SL-depleted roots as compared to wild-type shoots joined to wild-type roots. The phenotype of hetero-grafted plants is likely explained, mechanistically, by their shoot hypersensitivity to endogenous and exogenous ABA, in terms of stomatal closure. This, in turn, might be due to the increased SL levels in shoots as suggested by the up-regulation of SL-biosynthetic genes; treatment with exogenous SLs is indeed sufficient to increase sensitivity to ABA (Visentin et al., 2016). In the roots, the SL-ABA interactions might be different than in shoots: keeping SL levels high by a pre-treatment with exogenous SLs hampers the osmotic stress-triggered ABA increase in Lotus roots. Notwithstanding that such peculiar SL-ABA relationship should be verified in other models, this means that SLs repress ABA synthesis in the roots and thus, that their levels must drop also to allow local ABA build-up upon stress. This in turn may be needed both for local and systemic stress signaling, assuming shootward transport of ABA (Liu et al., 2015). It must be noted here that while it is known that shootward translocation of rootsynthesized ABA is important for systemic signaling of stress in some plant species (Manzi et al., 2015), it is not in all plants. Tomato shoots, for example, do not need root-produced ABA in order to react appropriately to water scarcity in soil (Chen et al., 2002).

\section{CONCLUSION AND FUTURE PROSPECTIVE}

The SL-ABA cross-talk has been increasingly spotlighted by research aimed at dissecting and understanding abiotic stress tolerance; interest is escalating because of the theoretical possibility of engineering phytohormonal signaling and metabolism for the improvement of crop performances under natural stress conditions. If we really are to do so, and exploit SL biology to the purpose of sustainable agriculture, the most immediate challenge ahead is to understand the molecular underpinnings of the pervasive SL effects on plant phenotypes under stress, as well as how they may connect physiological strain to appropriate development progression. How do SLs modulate ABA sensitivity? Is their effect linked to altered efficiency or abundance of components in the ABA signaling machinery, and/or to a modulation of ABA transport? Data in Arabidopsis, Lotus and tomato indicate that in the former, ABA transporters are down-regulated in SL-depleted vs. wild-type leaves under drought (Ha et al., 2014); while in the latter two, SL-defective leaves retain the ability to close their stomata comparably to the wild-type in response to exogenous ABA fed through the petiole, if given sufficient time (Liu et al., 2015; Visentin et al., 2016). Both observations support some contribution to the final phenotype of SL-depleted plants, for less efficient ABA translocation. Also, is the effect on sensitivity mutual, i.e., is ABA able to affect sensitivity to SLs, and/or their translocation? Is there a connection between SLs and regulatory molecules such as miRNAs (Marzec and Muszynska, 2015), as proven for other hormones (Curaba et al., 2014; Ferdous et al., 2015) and if so, is this connection relevant for SL effects under drought? Finally, based on the newly acquired knowledge, can we envisage breeding or management strategies that can move agriculture one step closer to sustainability, for example by exploiting the drought-tolerant phenotype of heterografted tomato plants? As reported by Visentin et al. (2016).

\section{AUTHOR CONTRIBUTIONS}

WS has compiled the literature and written the manuscript. ZA has initiated and supervised the research. ZA and SN have helped in compilation of manuscript. All three authors have edited and revised the approved manuscript submitted. WS and SN contributed equally in this manuscript.

\section{ACKNOWLEDGMENTS}

WS is thankful to Higher Education Commission of Pakistan for providing funding of research and Ph.D. Fellowship under Indigenous Ph.D. Fellowship Program 2BM2-61. All the authors are thankful to COMSATS Institute of Information Technology, Islamabad-Pakistan for providing the research infrastructure. 


\section{REFERENCES}

Abe, S., Sado, A., Tanaka, K., Kisugi, T., Asami, K., Ota, S., et al. (2014). Carlactone is converted to carlactonoic acid by MAX1 in Arabidopsis and its methyl ester can directly interact with AtD14 in vitro. Proc. Natl. Acad. Sci. U.S.A. 111, 18084-18089. doi: 10.1073/pnas.1410801111

Akiyama, K., Matsuzaki, K., and Hayashi, H. (2005). Plant sesquiterpenes induce hyphal branching in arbuscular mycorrhizal fungi. Nature 435, 824-827. doi: $10.1038 /$ nature 03608

Al-Babili, S., and Bouwmeester, H. J. (2015). Strigolactones, a novel carotenoidderived plant hormone. Annu. Rev. Plant Biol. 66, 161-186. doi: 10.1146/ annurev-arplant-043014-114759

Alder, A., Jamil, M., Marzorati, M., Bruno, M., Vermathen, M., Bigler, P., et al. (2012). The path from $\beta$-carotene to carlactone, a strigolactone-like plant hormone. Science 335, 1348-1351. doi: 10.1126/science.1218094

Arc, E., Sechet, J., Corbineau, F., Rajjou, L., and Marion-Poll, A. (2013). ABA crosstalk with ethylene and nitric oxide in seed dormancy and germination. Front. Plant Sci. 4:63. doi: 10.3389/fpls.2013.00063

Arite, T., Iwata, H., Ohshima, K., Maekawa, M., Nakajima, M., Kojima, M., et al. (2007). DWARF10, an RMS1/MAX4/DAD1 ortholog, controls lateral bud outgrowth in rice. Plant J. 51, 1019-1029. doi: 10.1111/j.1365-313X.2007. 03210.x

Arite, T., Umehara, M., Ishikawa, S., Hanada, A., Maekawa, M., Yamaguchi, S., et al. (2009). d14, a strigolactone-insensitive mutant of rice, shows an accelerated outgrowth of tillers. Plant Cell Physiol. 50, 1416-1424. doi: 10.1093/pcp/pcp091

Aroca, R., del Mar Alguacil, M., Vernieri, P., and Ruiz-Lozano, J. M. (2008). Plant responses to drought stress and exogenous $\mathrm{ABA}$ application are modulated differently by mycorrhization in tomato and an ABA-deficient mutant (Sitiens). Microb. Ecol. 56, 704-719. doi: 10.1007/s00248-008-9390-y

Aroca, R., Ruiz-Lozano, J. M., Zamarreño, ÁM., Paz, J. A., García-Mina, J. M., Pozo, M. J., et al. (2013). Arbuscular mycorrhizal symbiosis influences strigolactone production under salinity and alleviates salt stress in lettuce plants. J. Plant Physiol. 170, 47-55. doi: 10.1016/j.jplph.2012.08.020

Auldridge, M. E., McCarty, D. R., and Klee, H. J. (2006). Plant carotenoid cleavage oxygenases and their apocarotenoid products. Curr. Opin. Plant Biol. 9, 315-321. doi: 10.1016/j.pbi.2006.03.005

Bennett, T., and Leyser, O. (2014). Strigolactone signalling: standing on the shoulders of DWARFs. Curr. Opin. Plant Biol. 22, 7-13. doi: 10.1016/j.pbi.2014. 08.001

Bennett, T., Sieberer, T., Willett, B., Booker, J., Luschnig, C., and Leyser, O. (2006). The Arabidopsis MAX pathway controls shoot branching by regulating auxin transport. Curr. Biol. 16, 553-563. doi: 10.1016/j.cub.2006.01.058

Besserer, A., Puech-Pagès, V., Kiefer, P., Gomez-Roldan, V., Jauneau, A., Roy, S., et al. (2006). Strigolactones stimulate arbuscular mycorrhizal fungi by activating mitochondria. PLoS Biol. 4:e226. doi: 10.1371/journal.pbio.0040226

Bonneau, L., Huguet, S., Wipf, D., Pauly, N., and Truong, H.-N. (2013). Combined phosphate and nitrogen limitation generates a nutrient stress transcriptome favorable for arbuscular mycorrhizal symbiosis in Medicago truncatula. New Phytol. 199, 188-202. doi: 10.1111/nph.12234

Booker, J., Auldridge, M., Wills, S., McCarty, D., Klee, H., and Leyser, O. (2004). MAX3/CCD7 is a carotenoid cleavage dioxygenase required for the synthesis of a novel plant signaling molecule. Curr. Biol. 14, 1232-1238. doi: 10.1016/j.cub. 2004.06.061

Booker, J., Sieberer, T., Wright, W., Williamson, L., Willett, B., Stirnberg, P., et al. (2005). MAX1 encodes a cytochrome P450 family member that acts downstream of $\mathrm{MAX} 3 / 4$ to produce a carotenoid-derived branchinhibiting hormone. Dev. Cell 8, 443-449. doi: 10.1016/j.devcel.2005. 01.009

Boursiac, Y., Léran, S., Corratgé-Faillie, C., Gojon, A., Krouk, G., and Lacombe, B. (2013). ABA transport and transporters. Trends Plant Sci. 18, 325-333. doi: 10.1016/j.tplants.2013.01.007

Bouwmeester, H. J., Roux, C., Lopez-Raez, J. A., and Bécard, G. (2007). Rhizosphere communication of plants, parasitic plants and AM fungi. Trends Plant Sci. 12, 224-230. doi: 10.1016/j.tplants.2007.03.009

Brewer, P. B., Dun, E. A., Ferguson, B. J., Rameau, C., and Beveridge, C. A. (2009). Strigolactone acts downstream of auxin to regulate bud outgrowth in pea and Arabidopsis. Plant Physiol. 150, 482-493. doi: 10.1104/pp.108. 134783
Brewer, P. B., Yoneyama, K., Filardo, F., Meyers, E., Scaffidi, A., Frickey, T., et al. (2016). LATERAL BRANCHING OXIDOREDUCTASE acts in the final stages of strigolactone biosynthesis in Arabidopsis. Proc. Natl. Acad. Sci. U.S.A. 113, 6301-6306. doi: 10.1073/pnas.1601729113

Bu, Q., Lv, T., Shen, H., Luong, P., Wang, J., Wang, Z., et al. (2014). Regulation of drought tolerance by the F-box protein MAX2 in arabidopsis. Plant Physiol. 164, 424-439. doi: 10.1104/pp.113.226837

Bythell-Douglas, R., Rothfels, C. J., Stevenson, D. W. D., Graham, S. W., Wong, G. K.-S., Nelson, D. C., et al. (2017). The Complex Origins of Strigolactone Signalling in Land Plants. Available at: http://www.biorxiv.org/content/early/ 2017/01/25/102715 [accessed August 2, 2017].

Chandler, P. M., and Robertson, M. (2003). Gene Expression Regulated by Abscisic Acid and its Relation to Stress Tolerance. Available at: http://www.annualreviews.org/doi/abs/10.1146/annurev.pp.45.060194. 000553? journalCode=arplant. 2 [accessed December 9, 2014].

Chen, G., Lips, S. H., and Sagi, M. (2002). Biomass production, transpiration rate and endogenous abscisic acid levels in grafts of flacca and wild-type tomato (Lycopersicon esculentum). Funct. Plant Biol. 29, 1329-1335. doi: 10.1071/ PP01263

Cheng, X., Ruyter-Spira, C., and Bouwmeester, H. (2013). The interaction between strigolactones and other plant hormones in the regulation of plant development. Front. Plant Sci. 4:199. doi: 10.3389/fpls.2013.00199

Chevalier, F., Nieminen, K., Sánchez-Ferrero, J. C., Rodríguez, M. L., Chagoyen, M., Hardtke, C. S., et al. (2014). Strigolactone promotes degradation of DWARF14, an $\alpha / \beta$ hydrolase essential for strigolactone signaling in Arabidopsis. Plant Cell 26, 1134-1150. doi: 10.1105/tpc.114.122903

Conn, C. E., and Nelson, D. C. (2015). Evidence that KARRIKIN-INSENSITIVE2 (KAI2) receptors may perceive an unknown signal that is not karrikin or strigolactone. Front. Plant Sci. 6:1219. doi: 10.3389/fpls.2015.01219

Crawford, S., Shinohara, N., Sieberer, T., Williamson, L., George, G., Hepworth, J., et al. (2010). Strigolactones enhance competition between shoot branches by dampening auxin transport. Development 137, 2905-2913. doi: 10.1242/dev. 051987

Curaba, J., Singh, M. B., and Bhalla, P. L. (2014). miRNAs in the crosstalk between phytohormone signalling pathways. J. Exp. Bot. 65, 1425-1438. doi: 10.1093/ jxb/eru002

Cutler, A. J., and Krochko, J. E. (1999). Formation and breakdown of ABA. Trends Plant Sci. 4, 472-478. doi: 10.1016/S1360-1385(99)01497-1

Davies, W. J., Wilkinson, S., and Loveys, B. (2002). Stomatal control by chemical signalling and the exploitation of this mechanism to increase water use efficiency in agriculture. New Phytol. 153, 449-460. doi: 10.1046/j.0028-646X. 2001.00345.x

De Cuyper, C., Fromentin, J., Yocgo, R. E., De Keyser, A., Guillotin, B., Kunert, K., et al. (2015). From lateral root density to nodule number, the strigolactone analogue GR24 shapes the root architecture of Medicago truncatula. J. Exp. Bot. 66, 137-146. doi: 10.1093/jxb/eru404

de Saint Germain, A., Clavé, G., Badet-Denisot, M.-A., Pillot, J.-P., Cornu, D. Le Caer, J.-P., et al. (2016). An histidine covalent receptor and butenolide complex mediates strigolactone perception. Nat. Chem. Biol. 12, 787-794. doi: $10.1038 /$ nchembio. 2147

Dharmasiri, N., Dharmasiri, S., and Estelle, M. (2005). The F-box protein TIR1 is an auxin receptor. Nature 435, 441-445. doi: 10.1038/nature03543

Fahad, S., Hussain, S., Bano, A., Saud, S., Hassan, S., Shan, D., et al. (2015). Potential role of phytohormones and plant growth-promoting rhizobacteria in abiotic stresses: consequences for changing environment. Environ. Sci. Pollut. Res. 22, 4907-4921. doi: 10.1007/s11356-014-3754-2

Ferdous, J., Hussain, S. S., and Shi, B.-J. (2015). Role of microRNAs in plant drought tolerance. Plant Biotechnol. J. 13, 293-305. doi: 10.1111/pbi.12318

Flematti, G. G. R., Scaffidi, A., Waters, M. T. M., and Smith, S. S. M. (2016). Stereospecificity in strigolactone biosynthesis and perception. Planta 243, 1361-1373. doi: 10.1007/s00425-016-2523-5

Flematti, G. R., Ghisalberti, E. L., Dixon, K. W., and Trengove, R. D. (2004). A compound from smoke that promotes seed germination. Science 305:977. doi: $10.1126 /$ science. 1099944

Foo, E., Bullier, E., Goussot, M., Foucher, F., Rameau, C., and Beveridge, C. A. (2005). The branching gene RAMOSUS1 mediates interactions among two novel signals and auxin in pea. Plant Cell 17, 464-474. doi: 10.1105/tpc.104. 026716 
Foo, E., Yoneyama, K., Hugill, C., Quittenden, L. J., and Reid, J. B. (2013). Strigolactones: internal and external signals in plant symbioses? Plant Signal. Behav. 8:e23168. doi: 10.4161/psb.23168

Fujita, Y., Fujita, M., Shinozaki, K., and Yamaguchi-Shinozaki, K. (2011). ABAmediated transcriptional regulation in response to osmotic stress in plants. J. Plant Res. 124, 509-525. doi: 10.1007/s10265-011-0412-3

Gomez-Roldan, V., Fermas, S., Brewer, P. B., Puech-Pagès, V., Dun, E. A., Pillot, J.-P., et al. (2008). Strigolactone inhibition of shoot branching. Nature 455, 189-194. doi: 10.1038/nature07271

Gonzalez-Perez, S., Gutierrez, J., Garcia-Garcia, F., Osuna, D., Dopazo, J., Lorenzo, O., et al. (2011). Early transcriptional defense responses in Arabidopsis cell suspension culture under high-light conditions. Plant Physiol. 156, 14391456. doi: $10.1104 /$ pp.111.177766

Ha, C. V., Leyva-González, M. A., Osakabe, Y., Tran, U. T., Nishiyama, R., Watanabe, Y., et al. (2014). Positive regulatory role of strigolactone in plant responses to drought and salt stress. Proc. Natl. Acad. Sci. U.S.A. 111, 851-856. doi: 10.1073/pnas.1322135111

Hamiaux, C., Drummond, R. S. M., Janssen, B. J., Ledger, S. E., Cooney, J. M., Newcomb, R. D., et al. (2012). DAD2 is an $\alpha / \beta$ hydrolase likely to be involved in the perception of the plant branching hormone, strigolactone. Curr. Biol. 22, 2032-2036. doi: 10.1016/j.cub.2012.08.007

Hayward, A., Stirnberg, P., Beveridge, C., and Leyser, O. (2009). Interactions between auxin and strigolactone in shoot branching control. Plant Physiol. 151, 400-412. doi: 10.1104/pp.109.137646

Herde, O., Pena Cortes, H., Wasternack, Willmitzer, L., and Fisahn, J. (1999). Electric signaling and pin2 gene expression on different abiotic stimuli depend on a distinct threshold level of endogenous abscisic acid in several abscisic aciddeficient tomato mutants. Plant Physiol. 119, 213-218. doi: 10.1104/pp.119. 1.213

Herrera-Medina, M. J., Steinkellner, S., Vierheilig, H., Ocampo Bote, J. A., and García Garrido, J. M. (2007). Abscisic acid determines arbuscule development and functionality in the tomato arbuscular mycorrhiza. New Phytol. 175, 554-564. doi: 10.1111/j.1469-8137.2007.02107.x

Hong, J. H., Seah, S. W., and Xu, J. (2013). The root of ABA action in environmental stress response. Plant Cell Rep. 32, 971-983. doi: 10.1007/s00299-013-1439-9

Ito, S., Ito, K., Abeta, N., Takahashi, R., Sasaki, Y., and Yajima, S. (2016). Effects of strigolactone signaling on Arabidopsis growth under nitrogen deficient stress condition. Plant Signal. Behav. 11:e1126031. doi: 10.1080/15592324.2015. 1126031

Ito, S., Nozoye, T., Sasaki, E., Imai, M., Shiwa, Y., Shibata-Hatta, M., et al. (2015). Strigolactone regulates anthocyanin accumulation, acid phosphatases production and plant growth under low phosphate condition in Arabidopsis. PLoS ONE 10:e0119724. doi: 10.1371/journal.pone.0119724

Jamil, M., Charnikhova, T., Cardoso, C., Jamil, T., Ueno, K., Verstappen, F., et al. (2011). Quantification of the relationship between strigolactones and striga hermonthica infection in rice under varying levels of nitrogen and phosphorus. Weed Res. 51, 373-385. doi: 10.1111/j.1365-3180.2011.00847.x

Jamil, M., Charnikhova, T., Verstappen, F., Ali, Z., Wainwright, H., and Bouwmeester, H. J. (2014). Effect of phosphate-based seed priming on strigolactone production and Striga hermonthica infection in cereals. Weed Res. 54, 307-313. doi: $10.1111 /$ wre. 12067

Jia, K.-P., Luo, Q., He, S.-B., Lu, X.-D., and Yang, H.-Q. (2014). Strigolactoneregulated hypocotyl elongation is dependent on cryptochrome and phytochrome signaling pathways in Arabidopsis. Mol. Plant 7, 528-540. doi: $10.1093 / \mathrm{mp} / \mathrm{sst} 093$

Jiang, L., Liu, X., Xiong, G., Liu, H., Chen, F., Wang, L., et al. (2013). DWARF 53 acts as a repressor of strigolactone signalling in rice. Nature 504, 401-405. doi: 10.1038/nature12870

Johnson, X., Brcich, T., Dun, E. A., Goussot, M., Haurogné, K., Beveridge, C. A., et al. (2006). Branching genes are conserved across species. Genes controlling a novel signal in pea are coregulated by other long-distance signals. Plant Physiol. 142, 1014-1026. doi: 10.1104/pp.106.087676

Kapulnik, Y., Delaux, P.-M., Resnick, N., Mayzlish-Gati, E., Wininger, S., Bhattacharya, C., et al. (2011). Strigolactones affect lateral root formation and root-hair elongation in Arabidopsis. Planta 233, 209-216. doi: 10.1007/s00425010-1310-y

Kohlen, W., Charnikhova, T., Lammers, M., Pollina, T., Tóth, P., Haider, I., et al. (2012). The tomato CAROTENOID CLEAVAGE DIOXYGENASE8 (SICCD8) regulates rhizosphere signaling, plant architecture and affects reproductive development through strigolactone biosynthesis. New Phytol. 196, 535-547. doi: $10.1111 / j .1469-8137.2012 .04265 . x$

Koltai, H. (2015). Cellular events of strigolactone signalling and their crosstalk with auxin in roots. J. Exp. Bot. 66, 4855-4861. doi: 10.1093/jxb/erv178

Koltai, H., Dor, E., Hershenhorn, J., Joel, D. M., Weininger, S., Lekalla, S., et al. (2010). Strigolactones' effect on root growth and root-hair elongation may be mediated by auxin-efflux carriers. J. Plant Growth Regul. 29, 129-136. doi: 10.1007/s00344-009-9122-7

Koltai, H., and Prandi, C. (2016). Strigolactones: past, present and future. Planta 243, 1309-1309. doi: 10.1007/s00425-016-2541-3

Kretzschmar, T., Kohlen, W., Sasse, J., Borghi, L., Schlegel, M., Bachelier, J. B., et al. (2012). A petunia $A B C$ protein controls strigolactone-dependent symbiotic signalling and branching. Nature 483, 341-344. doi: 10.1038/nature10873

Linkohr, B. I., Williamson, L. C., Fitter, A. H., and Leyser, H. M. O. (2002). Nitrate and phosphate availability and distribution have different effects on root system architecture of Arabidopsis. Plant J. 29, 751-760. doi: 10.1046/j.1365-313X. 2002.01251.x

Liu, J., He, H., Vitali, M., Visentin, I., Charnikhova, T., Haider, I., et al. (2015). Osmotic stress represses strigolactone biosynthesis in Lotus japonicus roots: exploring the interaction between strigolactones and ABA under abiotic stress. Planta 241, 1435-1451. doi: 10.1007/s00425-015-2266-8

Liu, J., Lovisolo, C., Schubert, A., and Cardinale, F. (2013). Signaling role of Strigolactones at the interface between plants, (micro)organisms, and a changing environment. J. Plant Interact. 8, 17-33. doi: 10.1080/17429145.2012. 750692

López-Ráez, J. A. (2016). How drought and salinity affect arbuscular mycorrhizal symbiosis and strigolactone biosynthesis? Planta 243, 1375-1385. doi: 10.1007/ s00425-015-2435-9

López-Ráez, J. A., Charnikhova, T., Gómez-Roldán, V., Matusova, R., Kohlen, W., De Vos, R., et al. (2008a). Tomato strigolactones are derived from carotenoids and their biosynthesis is promoted by phosphate starvation. New Phytol. 178, 863-874. doi: 10.1111/j.1469-8137.2008.02406.x

López-Ráez, J. A., Charnikhova, T., Mulder, P., Kohlen, W., Bino, R., Levin, I., et al. (2008b). Susceptibility of the tomato mutant high pigment-2 ${ }^{d g}\left(h p-2^{d g}\right)$ to Orobanche spp. infection. J. Agric. Food Chem. 56, 6326-6332. doi: 10.1021/ jf800760x

López-Ráez, J. A., Kohlen, W., Charnikhova, T., Mulder, P., Undas, A. K., Sergeant, M. J., et al. (2010). Does abscisic acid affect strigolactone biosynthesis? New Phytol. 187, 343-354. doi: 10.1111/j.1469-8137.2010.03291.x

Manzi, M., Lado, J., Rodrigo, M. J., Zacarías, L., Arbona, V., and GómezCadenas, A. (2015). Root ABA accumulation in long-term water-stressed plants is sustained by hormone transport from aerial organs. Plant Cell Physiol. 56, 2457-2466. doi: 10.1093/pcp/pcv161

Martín-Rodríguez, J. A., Huertas, R., Ho-Plágaro, T., Ocampo, J. A., Tureèková, V., Tarkowská, D., et al. (2016). Gibberellin-abscisic acid balances during arbuscular mycorrhiza formation in tomato. Front. Plant Sci. 7:1273. doi: $10.3389 /$ fpls.2016.01273

Marzec, M. (2016). Perception and signaling of strigolactones. Front. Plant Sci. 7:1260. doi: $10.3389 /$ fpls.2016.01260

Marzec, M., and Muszynska, A. (2015). In silico analysis of the genes encoding proteins that are involved in the biosynthesis of the RMS/MAX/D pathway revealed new roles of strigolactones in plants. Int. J. Mol. Sci. 16, 6757-6782. doi: 10.3390/ijms16046757

Matusova, R., Rani, K., Verstappen, F. W. A., Franssen, M. C. R., Beale, M. H., and Bouwmeester, H. J. (2005). The strigolactone germination stimulants of the plant-parasitic Striga and Orobanche spp. are derived from the carotenoid pathway. Plant Physiol. 139, 920-934. doi: 10.1104/pp.105.061382

Mayzlish-Gati, E., De-Cuyper, C., Goormachtig, S., Beeckman, T., Vuylsteke, M., Brewer, P. B., et al. (2012). Strigolactones are involved in root response to low phosphate conditions in Arabidopsis. Plant Physiol. 160, 1329-1341. doi: $10.1104 /$ pp.112.202358

Merilo, E., Jalakas, P., Kollist, H., and Brosché, M. (2015). The role of ABA recycling and transporter proteins in rapid stomatal responses to reduced air humidity, elevated CO2, and exogenous ABA. Mol. Plant 8, 657-659. doi: 10.1016/j.molp. 2015.01.014

Morris, S. E., Turnbull, C. G., Murfet, I. C., and Beveridge, C. A. (2001). Mutational analysis of branching in pea. Evidence that Rms1 and Rms 5 regulate 
the same novel signal. Plant Physiol. 126, 1205-1213. doi: 10.1104/pp.126.3. 1205

Nakamura, H., Xue, Y.-L., Miyakawa, T., Hou, F., Qin, H.-M., Fukui, K., et al. (2013). Molecular mechanism of strigolactone perception by DWARF14. Nat. Commun. 4, 189-194. doi: 10.1038/ncomms3613

Nelson, D. C., Scaffidi, A., Dun, E. A., Waters, M. T., Flematti, G. R., Dixon, K. W., et al. (2011). F-box protein MAX2 has dual roles in karrikin and strigolactone signaling in Arabidopsis thaliana. Proc. Natl. Acad. Sci. U.S.A. 108, 8897-8902. doi: 10.1073/pnas.1100987108

Niu, Y. F., Chai, R. S., Jin, G. L., Wang, H., Tang, C. X., and Zhang, Y. S. (2013). Responses of root architecture development to low phosphorus availability: a review. Ann. Bot. 112, 391-408. doi: 10.1093/aob/mcs285

Péret, B., Clément, M., Nussaume, L., and Desnos, T. (2011). Root developmental adaptation to phosphate starvation: better safe than sorry. Trends Plant Sci. 16, 442-450. doi: 10.1016/j.tplants.2011.05.006

Péret, B., Desnos, T., Jost, R., Kanno, S., Berkowitz, O., and Nussaume, L. (2014). Root architecture responses: in search of phosphate. Plant Physiol. 166, 1713-1723. doi: 10.1104/pp.114.244541

Pérez-Torres, C.-A., López-Bucio, J., Cruz-Ramírez, A., Ibarra-Laclette, E., Dharmasiri, S., Estelle, M., et al. (2008). Phosphate availability alters lateral root development in Arabidopsis by modulating auxin sensitivity via a mechanism involving the TIR1 auxin receptor. Plant Cell 20, 3258-3272. doi: 10.1105/tpc. 108.058719

Prusinkiewicz, P., Crawford, S., Smith, R. S., Ljung, K., Bennett, T., Ongaro, V., et al. (2009). Control of bud activation by an auxin transport switch. Proc. Natl. Acad. Sci. U.S.A. 106, 17431-17436. doi: 10.1073/pnas.0906696106

Ruiz-Lozano, J. M., Aroca, R., Zamarreño, ÁM., Molina, S., Andreo-Jiménez, B., Porcel, R., et al. (2016). Arbuscular mycorrhizal symbiosis induces strigolactone biosynthesis under drought and improves drought tolerance in lettuce and tomato. Plant. Cell Environ. 39, 441-452. doi: 10.1111/pce. 12631

Ruyter-Spira, C., Al-Babili, S., van der Krol, S., and Bouwmeester, H. (2013). The biology of strigolactones. Trends Plant Sci. 18, 72-83. doi: 10.1016/j.tplants. 2012.10.003

Scaffidi, A., Waters, M. T., Bond, C. S., Dixon, K. W., Smith, S. M., Ghisalberti, E. L., et al. (2012). Exploring the molecular mechanism of karrikins and strigolactones. Bioorg. Med. Chem. Lett. 22, 3743-3746. doi: 10.1016/j.bmcl. 2012.04.016

Scaffidi, A., Waters, M. T., Ghisalberti, E. L., Dixon, K. W., Flematti, G. R., and Smith, S. M. (2013). Carlactone-independent seedling morphogenesis in Arabidopsis. Plant J. 76, 1-9. doi: 10.1111/tpj.12265

Schwartz, S. H., Qin, X., and Loewen, M. C. (2004). The biochemical characterization of two carotenoid cleavage enzymes from Arabidopsis indicates that a carotenoid-derived compound inhibits lateral branching. J. Biol. Chem. 279, 46940-46945. doi: 10.1074/jbc.M409004200

Seto, Y., Sado, A., Asami, K., Hanada, A., Umehara, M., Akiyama, K., et al. (2014). Carlactone is an endogenous biosynthetic precursor for strigolactones. Proc. Natl. Acad. Sci. U.S.A. 111, 1640-1645. doi: 10.1073/pnas.1314805111

Seto, Y., and Yamaguchi, S. (2014). Strigolactone biosynthesis and perception. Curr. Opin. Plant Biol. 21, 1-6. doi: 10.1016/j.pbi.2014.06.001

Smith, S. M., and Li, J. (2014). Signalling and responses to strigolactones and karrikins. Curr. Opin. Plant Biol. 21, 23-29. doi: 10.1016/j.pbi.2014.06.003

Snowden, K. C., Simkin, A. J., Janssen, B. J., Templeton, K. R., Loucas, H. M., Simons, J. L., et al. (2005). The decreased apical dominancel/Petunia hybrida CAROTENOID CLEAVAGE DIOXYGENASE8 gene affects branch production and plays a role in leaf senescence, root growth, and flower development. Plant Cell 17, 746-759. doi: 10.1105/tpc.104.027714

Sorefan, K., Booker, J., Haurogné, K., Goussot, M., Bainbridge, K., Foo, E., et al. (2003). MAX4 and RMS1 are orthologous dioxygenase-like genes that regulate shoot branching in Arabidopsis and pea. Genes Dev. 17, 1469-1474. doi: 10.1101/gad.256603

Soto, M. J., Fernández-Aparicio, M., Castellanos-Morales, V., García-Garrido, J. M., Ocampo, J. A., Delgado, M. J., et al. (2010). First indications for the involvement of strigolactones on nodule formation in alfalfa (Medicago sativa). Soil Biol. Biochem. 42, 383-385. doi: 10.1016/j.soilbio.2009.11.007

Soundappan, I., Bennett, T., Morffy, N., Liang, Y., Stanga, J. P., Abbas, A., et al. (2015). SMAX1-LIKE/D53 family members enable distinct MAX2-dependent responses to strigolactones and karrikins in Arabidopsis. Plant Cell 27, 3143-3159. doi: 10.1105/tpc.15.00562

Stanga, J. P., Smith, S. M., Briggs, W. R., and Nelson, D. C. (2013). SUPPRESSOR OF MORE AXILLARY GROWTH2 1 controls seed germination and seedling development in Arabidopsis. Plant Physiol. 163, 318-330. doi: 10.1104/pp.113. 221259

Stirnberg, P., Furner, I. J., and Ottoline Leyser, H. M. (2007). MAX2 participates in an SCF complex which acts locally at the node to suppress shoot branching. Plant J. 50, 80-94. doi: 10.1111/j.1365-313X.2007.03032.x

Stirnberg, P., van De Sande, K., and Leyser, H. M. O. (2002). MAX1 and MAX2 control shoot lateral branching in Arabidopsis. Development 129, 1131-1141. doi: $10.1105 /$ tpc.13.8.1779

Sun, H., Tao, J., Liu, S., Huang, S., Chen, S., Xie, X., et al. (2014). Strigolactones are involved in phosphate- and nitrate-deficiency-induced root development and auxin transport in rice. J. Exp. Bot. 65, 6735-6746. doi: 10.1093/jxb/eru029

Toh, S., Holbrook-Smith, D., Stokes, M. E., Tsuchiya, Y., and McCourt, P. (2014). Detection of parasitic plant suicide germination compounds using a highthroughput Arabidopsis HTL/KAI2 strigolactone perception system. Chem. Biol. 21, 988-998. doi: 10.1016/j.chembiol.2014.07.005

Tsuchiya, Y., Yoshimura, M., Sato, Y., Kuwata, K., Toh, S., Holbrook-Smith, D., et al. (2015). Probing strigolactone receptors in Striga hermonthica with fluorescence. Science 349, 864-868. doi: 10.1126/science.aab3831

Ueguchi-Tanaka, M., Nakajima, M., Motoyuki, A., and Matsuoka, M. (2007). Gibberellin receptor and its role in gibberellin signaling in plants. Annu. Rev. Plant Biol. 58, 183-198. doi: 10.1146/annurev.arplant.58.032806.103830

Umehara, M., Cao, M., Akiyama, K., Akatsu, T., Seto, Y., Hanada, A., et al. (2015). Structural requirements of Strigolactones for shoot branching inhibition in rice and arabidopsis. Plant Cell Physiol. 56, 1059-1072. doi: 10.1093/pcp/pcv028

Umehara, M., Hanada, A., Magome, H., Takeda-Kamiya, N., and Yamaguchi, S. (2010). Contribution of strigolactones to the inhibition of tiller bud outgrowth under phosphate deficiency in rice. Plant Cell Physiol. 51, 1118-1126. doi: $10.1093 / \mathrm{pcp} / \mathrm{pcq} 084$

Umehara, M., Hanada, A., Yoshida, S., Akiyama, K., Arite, T., Takeda-Kamiya, N., et al. (2008). Inhibition of shoot branching by new terpenoid plant hormones. Nature 455, 195-200. doi: 10.1038/nature07272

Visentin, I., Vitali, M., Ferrero, M., Zhang, Y., Ruyter-Spira, C., Novák, O., et al. (2016). Low levels of strigolactones in roots as a component of the systemic signal of drought stress in tomato. New Phytol. 212, 954-963. doi: 10.1111/nph. 14190

Waldie, T., McCulloch, H., and Leyser, O. (2014). Strigolactones and the control of plant development: lessons from shoot branching. Plant J. 79, 607-622. doi: $10.1111 /$ tpj.12488

Wallner, E.-S., López-Salmerón, V., and Greb, T. (2016). Strigolactone versus gibberellin signaling: reemerging concepts? Planta 243, 1339-1350. doi: 10.1007/s00425-016-2478-6

Wani, S. H., Kumar, V., Shriram, V., and Sah, S. K. (2016). Phytohormones and their metabolic engineering for abiotic stress tolerance in crop plants. Crop J. 4, 162-176. doi: 10.1016/j.cj.2016.01.010

Waters, M. T., Brewer, P. B., Bussell, J. D., Smith, S. M., and Beveridge, C. A. (2012a). The Arabidopsis ortholog of rice DWARF27 Acts upstream of MAX1 in the control of plant development by strigolactones. Plant Physiol. 159, 1073-1085. doi: 10.1104/pp.112.196253

Waters, M. T., Gutjahr, C., Bennett, T., and Nelson, D. C. (2017). Strigolactone signaling and evolution. Annu. Rev. Plant Biol. 68, 291-322. doi: 10.1146/ annurev-arplant-042916-040925

Waters, M. T., Scaffidi, A., Flematti, G. R., and Smith, S. M. (2012b). Karrikins force a rethink of strigolactone mode of action. Plant Signal. Behav. 7, 969-972. doi: $10.4161 /$ psb.20977

Wilkinson, S., and Davies, W. J. (2002). ABA-based chemical signalling: the coordination of responses to stress in plants. Plant, Cell Environ. 25, 195-210. doi: 10.1046/j.0016-8025.2001.00824.x

Xie, X. (2016). Structural diversity of strigolactones and their distribution in the plant kingdom. J. Pestic. Sci. 41, 175-180. doi: 10.1584/jpestics.J16-02

Xie, X., Yoneyama, K., and Yoneyama, K. (2010). The strigolactone story. Annu. Rev. Phytopathol. 48, 93-117. doi: 10.1146/annurev-phyto-073009-114453

Xu, J., Zha, M., Li, Y., Ding, Y., Chen, L., Ding, C., et al. (2015). The interaction between nitrogen availability and auxin, cytokinin, and strigolactone in the 
control of shoot branching in rice (Oryza sativa L.). Plant Cell Rep. 34, 1647-1662. doi: 10.1007/s00299-015-1815-8

Yoneyama, K., Xie, X., Kim, H., Kisugi, T., Nomura, T., Sekimoto, H., et al. (2012). How do nitrogen and phosphorus deficiencies affect strigolactone production and exudation? Planta 235, 1197-1207. doi: 10.1007/s00425-011$1568-8$

Yoneyama, K., Xie, X., Kisugi, T., Nomura, T., and Yoneyama, K. (2013). Nitrogen and phosphorus fertilization negatively affects strigolactone production and exudation in sorghum. Planta 238, 885-894. doi: 10.1007/s00425-0131943-8

Yoneyama, K., Xie, X., Kusumoto, D., Sekimoto, H., Sugimoto, Y., Takeuchi, Y., et al. (2007a). Nitrogen deficiency as well as phosphorus deficiency in sorghum promotes the production and exudation of 5-deoxystrigol, the host recognition signal for arbuscular mycorrhizal fungi and root parasites. Planta 227, 125-132. doi: 10.1007/s00425-007-0600-5

Yoneyama, K., Yoneyama, K., Takeuchi, Y., and Sekimoto, H. (2007b). Phosphorus deficiency in red clover promotes exudation of orobanchol, the signal for mycorrhizal symbionts and germination stimulant for root parasites. Planta 225, 1031-1038. doi: 10.1007/s00425-006-0410-1

Yoneyama, K., Xie, X., Sekimoto, H., Takeuchi, Y., Ogasawara, S., Akiyama, K., et al. (2008). Strigolactones, host recognition signals for root parasitic plants and arbuscular mycorrhizal fungi, from Fabaceae plants. New Phytol. 179, 484-494. doi: $10.1111 /$ j.1469-8137.2008.02462.x

Zeevaart, J. A. D., and Creelman, R. A. (1988). Metabolism and physiology of abscisic acid. Annu. Rev. Plant Physiol. Plant Mol. Biol. 39, 439-473. doi: 10.1146/annurev.pp.39.060188.002255

Zhang, Y., Haider, I., Ruyter-Spira, C., and Bouwmeester, H. J. (2013). "Strigolactone biosynthesis and biology," in Molecular Microbial Ecology of the
Rhizosphere, Vol. 1 and 2, ed. F. J. de Bruijn (Hoboken, NJ: John Wiley \& Sons, Inc.). doi: 10.1002/9781118297674.ch33

Zhang, Y., Ruyter-Spira, C., and Bouwmeester, H. J. (2015). Engineering the plant rhizosphere. Curr. Opin. Biotechnol. 32, 136-142. doi: 10.1016/j.copbio.2014. 12.006

Zhang, Y., van Dijk, A. D. J., Scaffidi, A., Flematti, G. R., Hofmann, M., Charnikhova, T., et al. (2014). Rice cytochrome P450 MAX1 homologs catalyze distinct steps in strigolactone biosynthesis. Nat. Chem. Biol. 10, 1028-1033. doi: $10.1038 /$ nchembio. 1660

Zhao, L.-H., Zhou, X. E., Wu, Z.-S., Yi, W., Xu, Y., Li, S., et al. (2013). Crystal structures of two phytohormone signal-transducing $\alpha / \beta$ hydrolases: karrikinsignaling KAI2 and strigolactone-signaling DWARF14. Cell Res. 23, 436-439. doi: $10.1038 / \mathrm{cr} .2013 .19$

Zhao, L.-H., Zhou, X. E., Yi, W., Wu, Z., Liu, Y., Kang, Y., et al. (2015). Destabilization of strigolactone receptor DWARF14 by binding of ligand and E3-ligase signaling effector DWARF3. Cell Res. 25, 1219-1236. doi: 10.1038/cr. 2015.122

Conflict of Interest Statement: The authors declare that the research was conducted in the absence of any commercial or financial relationships that could be construed as a potential conflict of interest.

Copyright $\odot 2017$ Saeed, Naseem and Ali. This is an open-access article distributed under the terms of the Creative Commons Attribution License (CC BY). The use, distribution or reproduction in other forums is permitted, provided the original author(s) or licensor are credited and that the original publication in this journal is cited, in accordance with accepted academic practice. No use, distribution or reproduction is permitted which does not comply with these terms. 